\title{
The optimal time of initiation of renal replacement therapy in acute kidney injury: A meta-analysis
}

\author{
Kaiping Luo ${ }^{1, *}$, Shufang $\mathrm{Fu}^{1,{ }^{1}}$, Weidong Fang ${ }^{2}$ and Gaosi $\mathrm{Xu}{ }^{1}$ \\ ${ }^{1}$ Department of Nephrology, The Second Affiliated Hospital of Nanchang University, Nanchang, China \\ ${ }^{2}$ Department of Nephrology, People's Hospital of Ganzhou, Ganzhou, China \\ *These authors have contributed equally to this work \\ Correspondence to: Gaosi Xu, email: gaosixu@163.com \\ Keywords: acute kidney injury, renal replacement therapy, mortality, meta-analysis \\ Received: January 09, $2017 \quad$ Accepted: March 30, $2017 \quad$ Published: May 16, 2017 \\ Copyright: Luo et al. This is an open-access article distributed under the terms of the Creative Commons Attribution License \\ 3.0 (CC BY 3.0), which permits unrestricted use, distribution, and reproduction in any medium, provided the original author and \\ source are credited.
}

\section{ABSTRACT}

Background: The impact on the timing of renal replacement therapy (RRT) initiation on clinical outcomes for patients with acute kidney injury (AKI) remains controversial.

Materials and methods: We searched the Cochrane Library, EMBASE, Global Health, MEDLINE, PubMed, the International Clinical Trials Registry Platform, and Web of Science.

Results: We included 49 studies involving 9698 patients. Pooled analysis of 5408 critically ill patients with AKI showed that early RRT was significantly associated with reduced mortality compared to late RRT [odds ratio (OR), 0.40; $95 \%$ confidential intervals (CI), $0.32-0.48 ; I^{2}, 50.2 \%$ ]. For 4290 non-critically ill patients with AKI, there was no statistically significant difference in the risk of mortality between early and late RRT (OR, 1.07; 95\% CI, $0.79-1.45 ; I^{2}, 73.0 \%$ ). Early RRT was markedly associated with shortened intensive care units (ICU) length of stay (LOS) and hospital LOS compared to late RRT in both critically ill and noncritically ill patients with AKI.

Conclusions: Early RRT probably reduce the mortality, ICU and hospital LOS in critically ill patients with AKI. Inversely, early RRT in non-critically ill patients with AKI did not decrease the mortality, but shortened the ICU and hospital LOS.

\section{INTRODUCTION}

Acute kidney injury (AKI) is increasingly common and associated with adverse clinical outcomes, including excess mortality and morbidity, and prolonged hospital length of stay (LOS) [1-4]. Renal replacement therapy (RRT) is the cornerstone for the treatment of severe AKI. Although RRT provokes a considerable escalation in the complexity of therapy, the optimal timing of initiation of RRT in patients with AKI has been the focus of those debates $[5,6]$. Conflicting results from clinical trials and systematic reviews have not resolved the debates, leaving clinicians to select the timing of initiation of RRT based on suboptimal evidence.
Studies aimed at determining the optimal time for starting RRT have evaluated the various arbitrary cutoffs for time from Intensive Care Unit (ICU) admission [7-9] or development of a biochemical "start time" [10, 11], AKI stage [12, 13], serum urea [14, 15], urine output $[16,17]$, fluid balance [18], and serum creatinine $[15,19$, 20]. However, the arbitrary cut-offs often differentiated between early and late RRT. Some data suggested that early compared with late RRT reduced the mortality with better renal recovery. Early initiation of RRT may produce benefits by avoiding hypervolemia, eliminating of uremic toxins, establishing acid-base homeostasis, and preventing other complications such as gastric hemorrhage and metabolic encephalopathy $[7,13,16]$. Late RRT may 
allow time for the stabilization of a patient's condition before RRT and may even avoid the RRT [12, 21-23]. Gaudry et al. showed that the mortality was lower in patients who never received RRT than those received RRT early or late $(37.1 \%$ vs. $48.5 \%$ or $61.8 \%)$, and the patients with late RRT were the most severely ill at baseline [13]. Thus, we hypothesized that the different severity of illness for patients with AKI who received early RRT may produce distinct effects on mortality. Therefore, we firstly performed a meta-analysis according to the severity of illness for patients with AKI to investigate the opportunity of RRT initiation.

3 earlier meta-analyses (Seabra et al. [24] identified 23 studies, Karvellas et al. [25] identified 15 studies and Wang et al. [26] included 51 trials) showed that early RRT could confer a survival benefit. 11 trials performed before 1985 in Seabra et al. and Wang et al. were excluded, and the addition of 10 recently published studies have been included in the present meta-analysis. However, a recent meta-analysis found no significant difference in mortality between early and late RRT [27], but included only nine "high-quality" studies. Furthermore, the included studies were limited with high heterogeneity. In the present study, we firstly made a definition of early RRT based on timebased cutoffs for patients with AKI to investigate the optimal timing of initiation of RRT.

\section{RESULTS}

\section{Study enrolment and characteristics}

Figure 1 outlines the process for study selection. 49 studies including 9 RCTs [10, 12, 13, 15, 16, 19, 21 23] and 40 observational studies [7-9, 11, 14, 17, 18, 20, 28-59] were included in our meta-analysis. The eligible studies were conducted from 1985 to 2016 with 9698 patients evaluated the timing of initiation of RRT in patients with AKI. The characteristics of the articles were listed in Table 1, and the details of risk of bias for RCTs were showed in Figure 2.

\section{Meta-analysis results}

\section{Primary outcomes}

Pooled analysis of 5408 critically ill patients with AKI showed that early RRT was markedly associated with reduced mortality compared to late RRT (OR, 0.40; $95 \%$ CI, $0.32-0.48 ; I^{2}, 50.2 \%$, Figure 3). For 4290 noncritically ill patients with AKI, there was no statistically significant difference in the risk of mortality between early and late RRT (OR, 1.07; 95\% CI, 0.79 - 1.45; $I^{2}, 73.0 \%$, Figure 3).

Subgroup analysis of critically ill patients was firstly conducted in the present study by using the definition of early according to time criteria versus biochemical indicators. The significant association between early RRT and reduced mortality was also found under the studies that defined early by time criteria [early RRT within 12 hours (OR, 0.28; 95\% CI, $0.16-0.49 ; I^{2}, 44.8 \%$ ), within 24 hours (OR, $0.37 ; 95 \%$ CI, $0.25-0.54 ; I^{2}, 0.0 \%$ ), within 48 hours (OR, $0.55 ; 95 \%$ CI, $0.39-0.77 ; I^{2}, 30.8 \%$ ), within 72 hours (OR, $0.45 ; 95 \% \mathrm{CI}, 0.29-0.69 ; I^{2}, 48.2 \%$ ), and after 72 hours (OR, 0.32; 95\% CI, $0.14-0.74 ; I^{2}, 71.4 \%$ )], and by biochemical parameters (OR, 0.40; 95\% CI, 0.25 - $0.64 ; I^{2}, 58.9 \%$ ). Subgroup analysis of non-critically ill patients depending on the definition of early showed no significant subgroup survival benefits from early RRT.

Subgroup analysis of critically ill patients was based on the type of ICU admission. Early RRT was significantly associated with reduced mortality compared to late RRT among surgical group (OR, 0.33; 95\% CI, $0.22-0.48 ; I^{2}$, $47.9 \%$ ) and mixed group (OR, 0.43; 95\% CI, $0.34-0.54$; $I^{2}, 49.8 \%$ ). Subgroup analysis of non-critically ill patients based on ICU admission type showed no evidence of survival advantage in early RRT.

Subgroup analysis of critically ill patients was also performed according to RRT modality [continuous renal replacement therapy (CRRT), intermittent hemodialysis (IHD) or Mixed]. We found a markedly significant reduce in mortality in critically ill patients assigned to early RRT in the CRRT group (OR, 0.40; 95\% CI, $0.30-0.54 ; I^{2}$, $28.4 \%$ ), IHD group (OR, 0.11; 95\% CI, $0.03-0.43 ; I^{2}$, $56.9 \%$ ) and Mixed group (OR, 0.45; 95\% CI, 0.35 - 0.57; $I^{2}, 53.6 \%$ ) when compared to late RRT. Subgroup analysis of non-critically ill patients according to RRT modality showed that early RRT could not confer a survival benefit (Table 2).

\section{Secondary outcomes}

For critically ill patients with AKI, as showed in Table 2, early RRT significantly shortened ICU (MD, $-0.41 ; 95 \%$ CI, -0.55 to $-0.27 ; I^{2}, 87.0 \%$ ) and hospital LOS (MD, $-0.36 ; 95 \%$ CI, -0.51 to $-0.20 ; I^{2}, 94.7 \%$ ) compared to late RRT. Similar results were obtained in non-critically ill patients with AKI in ICU (MD, $-1.47 ; 95 \% \mathrm{CI},-1.71$ to $-1.22 ; I^{2}, 89.3 \%$ ) and hospital LOS (MD, $-1.07 ; 95 \% \mathrm{CI}$, -1.31 to $\left.-0.82 ; I^{2}, 0 \%\right)$.

\section{Sensitivity, meta-regression analyses}

Statistically similar results were obtained after omitting each study of critically ill patients with AKI, and the results of the sensitivity analyses were robust. Sensitivity analyses showed that Elsevivrs et al. [20] was the main source of heterogeneity for the studies of noncritically ill patients with AKI, and the heterogeneity was significantly decreased by omitting the study. For noncritically ill patients with AKI, there was no statistically significant difference in the risk of mortality between early 


\section{Potentially relevant studies identified \\ 5316 Identified through database search \\ 43 Identified through other sources}

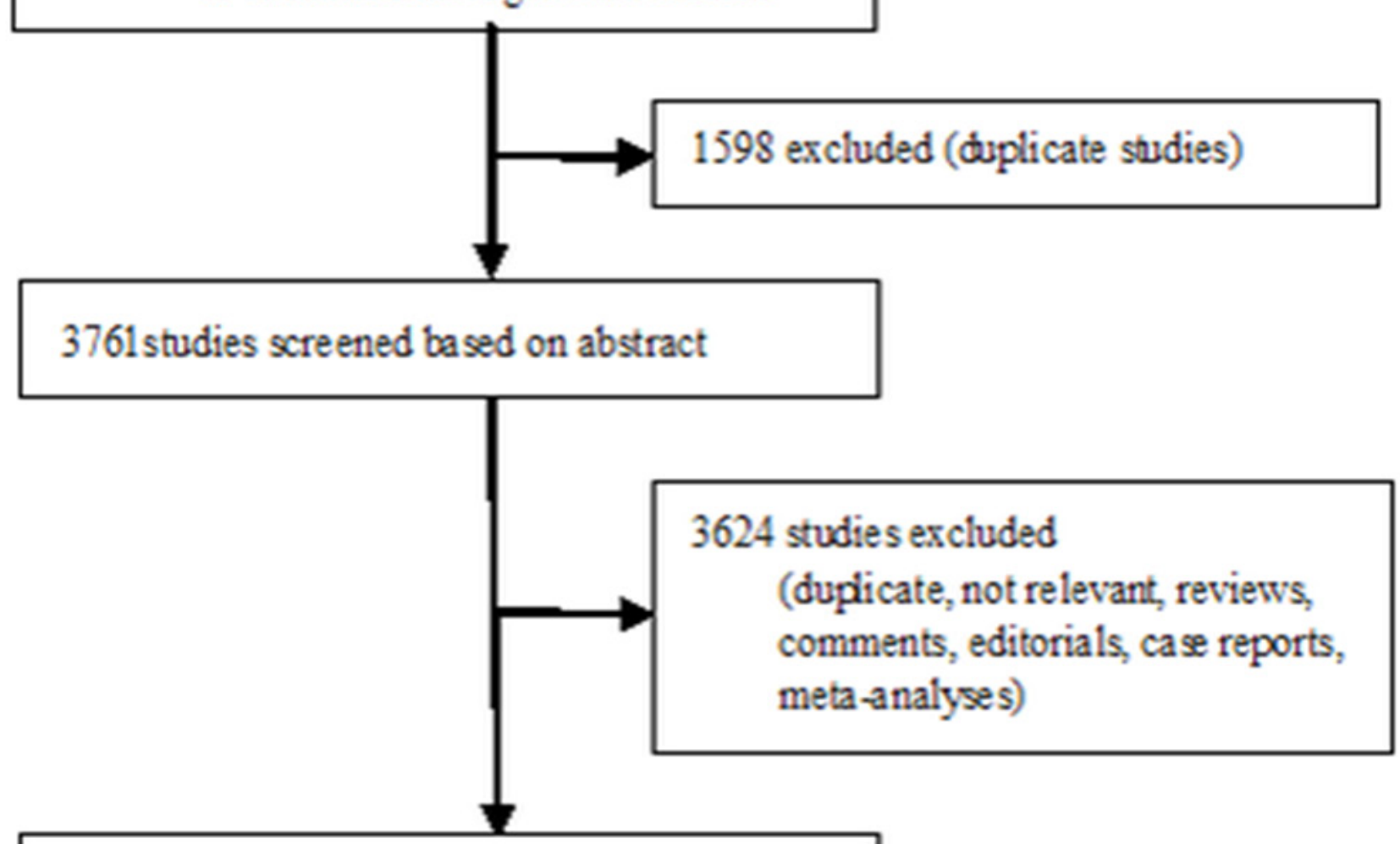

\section{7 full-text studies assessed for eligibility}

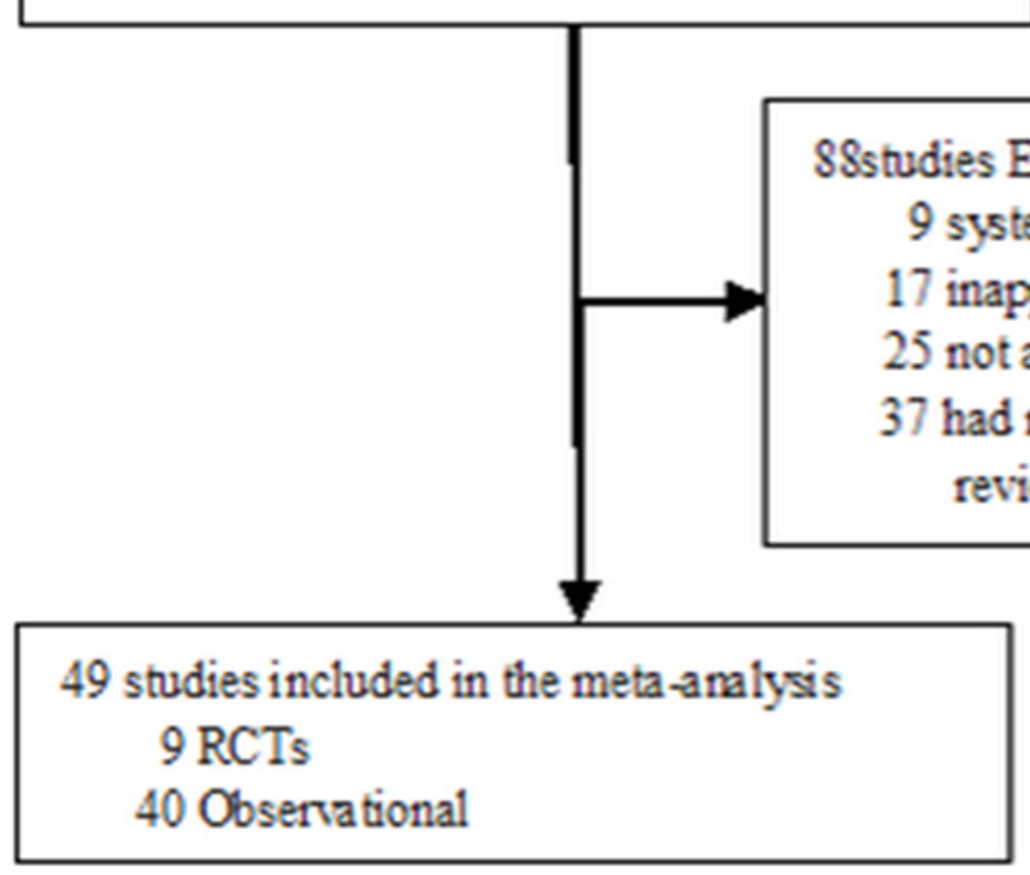

Figure 1: Flow diagram for the selection of studies inclusion in the meta-analysis. 
Table 1: The fundamental characteristics and patient demographic data of included studies reporting data on early RRT versus late RRT

\begin{tabular}{|c|c|c|c|c|c|c|c|c|c|}
\hline $\begin{array}{l}\text { Auther, } \\
\text { Year }\end{array}$ & Country & Study Design & Population & $\begin{array}{c}\text { Early } \\
\text { Mortality }\end{array}$ & $\begin{array}{c}\text { Late } \\
\text { Mortality }\end{array}$ & $\begin{array}{l}\text { Severity of } \\
\text { Illness }\end{array}$ & Early RRT Criteria & Late RRT Criteria & Quality \\
\hline \multicolumn{10}{|c|}{ Early time to RRT $<12 \mathrm{~h}$} \\
\hline $\begin{array}{l}\text { Bouman } \\
2002\end{array}$ & Netherlands & RCT & Multisystem & $20 / 70$ & $9 / 36$ & $\begin{array}{l}\text { Early: SOFA } 10.3 \\
\text { Late: SOFA } 10.6\end{array}$ & Time to RRT $<12 \mathrm{~h}$ & Time to RRT $>12 \mathrm{~h}$ & M \\
\hline $\begin{array}{l}\text { Piccinni } \\
2006\end{array}$ & Italy & Retrospective & Sepsis; ICU & $18 / 40$ & $29 / 40$ & $\begin{array}{l}\text { Early: APACHE } 2=27.2 ; \\
\text { Late: APACHE } 2=27.8\end{array}$ & Time to RRT $<12 \mathrm{~h}$ & No RRT & 7 \\
\hline $\begin{array}{l}\text { Andrade } \\
2007\end{array}$ & Brazil & Retrospective & $\begin{array}{l}\text { Multisystem; } \\
\text { Leptospirosis }\end{array}$ & $3 / 18$ & $10 / 15$ & $\begin{array}{l}\text { Early: APACHE } 2=24.5 \\
\text { Late: } \text { APACHE } 2=26\end{array}$ & $\begin{array}{l}\text { Mean time to RRT }= \\
\text { 4.4hrs }\end{array}$ & Mean time to RRT $=27.3 \mathrm{hrs}$ & 5 \\
\hline $\begin{array}{l}\text { Wu VC } \\
2007\end{array}$ & China & Retrospective & $\begin{array}{l}\text { Acute Liver } \\
\text { Failure; } \\
\text { Surgical ICU }\end{array}$ & $34 / 54$ & $22 / 26$ & $\begin{array}{l}\text { Early: } \mathrm{APACHE} 2=18 \\
\text { Late: } \mathrm{APACHE} 2=19\end{array}$ & $\begin{array}{l}\text { Mean time from ICU } \\
\text { admit to } \mathrm{RRT}=4.4 \mathrm{hrs} \text {; } \\
\mathrm{BUN}<80 \mathrm{mg} / \mathrm{dL} \text { AND } \\
\text { traditional indications } \\
\text { present }\end{array}$ & $\begin{array}{l}\text { Mean time from ICU admit to RRT } \\
=11.1 \mathrm{hrs} ; \mathrm{BUN}>80 \mathrm{mg} / \mathrm{dL} \text { AND } \\
\text { traditional indications present }\end{array}$ & 6 \\
\hline $\begin{array}{l}\text { Manche } \\
2008\end{array}$ & Malta & Retrospective & $\begin{array}{l}\text { Post Cardiac } \\
\text { Surgery }\end{array}$ & $14 / 56$ & $13 / 15$ & NR & $\begin{array}{l}\text { Mean RRT start } 8.6 \mathrm{hrs} \\
\text { post-op; Oliguria } \\
\text { unresponsive to med } \\
\text { mgmt }\end{array}$ & $\begin{array}{l}\text { Mean RRT start } 41.2 \mathrm{hrs} \text { post-op; } \\
\text { Oliguria refractory to med mgmt }\end{array}$ & 6 \\
\hline $\begin{array}{l}\text { Ji } \\
2011\end{array}$ & China & Retrospective & $\begin{array}{l}\text { Post Cardiac } \\
\text { Surgery }\end{array}$ & $3 / 34$ & $9 / 24$ & $\begin{array}{l}\text { Early: } \text { APACHE } 3=69 ; \\
\text { Late: APACHE3= } 88.2 \\
\text { p }<0.001\end{array}$ & $\begin{array}{l}\text { Time from urine output } \\
<0.5 \mathrm{ml} / \mathrm{kg} / \mathrm{h} \text { to } \mathrm{RRT}<12 \mathrm{~h} \text {; } \\
\text { Mean oliguria to start of } \\
\text { RRT } 8.4 \mathrm{hrs}\end{array}$ & $\begin{array}{l}\text { Time from urine output }<0.5 \mathrm{ml} / \mathrm{kg} / \mathrm{h} \\
\text { to RRT }>12 \mathrm{~h} \text {; Mean oliguria to start of } \\
\text { RRT21.5hrs }\end{array}$ & 6 \\
\hline $\begin{array}{l}\text { Shum } \\
2013\end{array}$ & China & Retrospective & $\begin{array}{l}\text { Multisystem; } \\
\text { Sepsis }\end{array}$ & $43 / 89$ & $15 / 31$ & $\begin{array}{l}\text { Early: SOFA 13; } \\
\text { Late: SOFA } 12 \\
\mathrm{P}=0.011\end{array}$ & $\begin{array}{l}\text { Mean time from ICU } \\
\text { admit to RRT } \\
=10.8 \mathrm{hrs} \text { (RIFLE criteria: } \\
\text { 'Injury' or 'Failure' } \\
\text { criteria) }\end{array}$ & $\begin{array}{l}\text { Mean time from ICU admit to RRT } \\
=20.7 \mathrm{hrs} \text { (RIFLE criteria: } \\
\text { 'pre- Risk' or 'Risk' criteria) }\end{array}$ & 6 \\
\hline $\begin{array}{l}\text { Serpytis } \\
2014\end{array}$ & Lithuania & Retrospective & $\begin{array}{l}\text { Multisystem; } \\
\text { Sepsis }\end{array}$ & $30 / 42$ & $39 / 43$ & NR & $\begin{array}{l}\text { Time from anuria to RRT } \\
<12 \mathrm{hrs}\end{array}$ & Time from anuria to $\mathrm{RRT}>12 \mathrm{hrs}$ & 5 \\
\hline $\begin{array}{l}\text { Wald } \\
2015\end{array}$ & Canada & RCT & Multisystem & $16 / 48$ & $19 / 52$ & $\begin{array}{l}\text { Early: SOFA } 13.3 \\
\text { Late: SOFA } 12.8\end{array}$ & $\begin{array}{l}\text { Mean time to RRT }= \\
9.7 \mathrm{hrs}\end{array}$ & $\begin{array}{l}\text { Meantime to RRT }=32 \mathrm{hrs} \\
\text { Classic indications for RRT }\end{array}$ & $\mathrm{H}$ \\
\hline $\begin{array}{l}\text { Crescenzi } \\
2015\end{array}$ & Italy & Prospective & $\begin{array}{l}\text { Post Cardiac } \\
\text { Surgery }\end{array}$ & $28 / 46$ & $10 / 13$ & NR & $\begin{array}{l}\text { Time from urine output } \\
<0.5 \mathrm{ml} / \mathrm{kg} / \mathrm{h} \\
\text { to } \mathrm{RRT}<12 \mathrm{~h}\end{array}$ & $\begin{array}{l}\text { Time from urine output }<0.5 \mathrm{ml} / \mathrm{kg} / \mathrm{h} \text { to } \\
\text { RRT }>12 \mathrm{~h}\end{array}$ & 6 \\
\hline $\begin{array}{l}\text { Zarbock } \\
2015\end{array}$ & Germany & RCT & Multisystem & $44 / 112$ & $65 / 119$ & $\begin{array}{l}\text { Early: SOFA } 15.6 \\
\text { Late: SOFA } 16.0\end{array}$ & $\begin{array}{l}\text { Time to RRT }<8 \mathrm{~h} \\
\text { KDIGO stage } 2\end{array}$ & $\begin{array}{l}\text { Time to RRT }<12 \mathrm{~h} \text {; Stage } 3 \mathrm{AKI} \\
\text { or no initiation }\end{array}$ & $\mathrm{H}$ \\
\hline $\begin{array}{l}\text { Gaudry } \\
2015\end{array}$ & France & RCT & Multisystem & $150 / 311$ & $153 / 308$ & $\begin{array}{l}\text { Early: SOFA } 10.9 \\
\text { Late: SOFA } 10.8\end{array}$ & $\begin{array}{l}\text { Time to RRT }<6 \mathrm{~h} \text {; Stage } \\
3 \text { AKI }\end{array}$ & $\begin{array}{l}\text { Classic indications for RRT; Oliguria or } \\
\text { anuria }>72 \mathrm{hrs} \text { after randomization }\end{array}$ & $\mathrm{H}$ \\
\hline \multicolumn{10}{|c|}{ Early time to RRT $<24 \mathrm{~h}$} \\
\hline $\begin{array}{l}\text { Elahi } \\
2004\end{array}$ & UK & Retrospective & $\begin{array}{l}\text { Post Cardiac } \\
\text { surgery }\end{array}$ & $8 / 36$ & $12 / 28$ & NR & $\begin{array}{l}\text { Mean RRT start } 0.78 \\
\text { days; } \\
\text { Low urine output }<100 \mathrm{ml} \\
\text { within } 8 \mathrm{~h} \text { after surgery }\end{array}$ & $\begin{array}{l}\text { Mean RRT start } 2.5 \text { days; Traditional } \\
\text { indications: Urea } \geq 30 \mathrm{mmol} / \mathrm{L} \text {, } \\
\mathrm{Cr} \geq 250 \mathrm{mmol} / \mathrm{L}, \mathrm{K}>6.0 \mathrm{mEq} / \mathrm{L}\end{array}$ & 6 \\
\hline $\begin{array}{l}\text { Demirkilic } \\
2004\end{array}$ & Turkey & Retrospective & $\begin{array}{l}\text { Post Cardiac } \\
\text { Surgery }\end{array}$ & $8 / 34$ & $15 / 27$ & NR & $\begin{array}{l}\text { Mean RRT start } 0.88 \\
\text { days; } \\
\text { Low urine output }<100 \mathrm{ml} \\
\text { within } 8 \text { hrs post-op; }\end{array}$ & $\begin{array}{l}\text { Mean RRT start } 2.56 \text { days; } \\
\mathrm{Cr} \geq 5 \mathrm{mg} / \mathrm{dL} \text {, or } \mathrm{K}>5.5 \mathrm{mEq} / \mathrm{L}\end{array}$ & 6 \\
\hline $\begin{array}{l}\text { Boussekey } \\
2012\end{array}$ & France & Retrospective & Multisystem & $28 / 67$ & $28 / 43$ & $\begin{array}{l}\text { Early: SOFA: } 11.1 ; \\
\text { Late: SOFA } 8.8 ; \\
\mathrm{p}=0.002\end{array}$ & $\begin{array}{l}\text { Time from RIFLE- } \\
\text { 'Injury' to RRT } \\
<16 \mathrm{hrs} \text {; Mean time to } \\
\text { RRT=6hrs }\end{array}$ & $\begin{array}{l}\text { Time from RIFLE- 'Injury' to RRT > } \\
\text { 16hrs; Mean time to RRT=64hrs }\end{array}$ & 7 \\
\hline $\begin{array}{l}\text { Chon } \\
2012\end{array}$ & Korea & Retrospective & $\begin{array}{l}\text { Multisystem; } \\
\text { Sepsis }\end{array}$ & $7 / 36$ & $9 / 19$ & $\begin{array}{l}\text { Early: SOFA } 13.5 \\
\text { Late: SOFA } 12\end{array}$ & $\begin{array}{l}\text { Time to RIFLE 'Injury'/ } \\
\text { 'Failure' } \\
<24 \mathrm{hrs} \text {; Mean time to } \\
\text { RRT=12.5hrs }\end{array}$ & $\begin{array}{l}\text { Time to RIFLE 'Injury'/ 'Failure' } \\
>24 \mathrm{hrs} \text {; Mean time to RRT }=42.2 \mathrm{hrs}\end{array}$ & 7 \\
\hline $\begin{array}{l}\text { Leite } \\
2013\end{array}$ & Brazil & Retrospective & Multisystem & $33 / 64$ & $67 / 86$ & $\begin{array}{l}\text { Early: } A P A C H E 2=19.2 ; \\
\text { Late: } A P A C H E 2=18.7\end{array}$ & $\begin{array}{l}\text { Time from AKIN } 3 \\
\text { diagnosis to RRT }<24 \mathrm{hrs}\end{array}$ & $\begin{array}{l}\text { Time from AKIN } 3 \text { diagnosis to RRT } \\
>24 \mathrm{hrs}\end{array}$ & 7 \\
\hline
\end{tabular}

(Continued) 


\begin{tabular}{|c|c|c|c|c|c|c|c|c|c|}
\hline $\begin{array}{l}\text { Auther, } \\
\text { Year }\end{array}$ & Country & Study Design & Population & $\begin{array}{c}\text { Early } \\
\text { Mortality }\end{array}$ & $\begin{array}{c}\text { Late } \\
\text { Mortality }\end{array}$ & $\begin{array}{l}\text { Severity of } \\
\text { Illness }\end{array}$ & Early RRT Criteria & Late RRT Criteria & Quality \\
\hline $\begin{array}{l}\text { Jun } \\
2014\end{array}$ & Australia & Prospective & $\begin{array}{l}\text { Multisystem; } \\
\text { Sepsis }\end{array}$ & $82 / 219$ & $84 / 220$ & $\begin{array}{l}\text { Early: SOFA: } 2.0 \\
\text { Late: SOFA } 2.1\end{array}$ & $\begin{array}{l}\text { Time from AKI diagnosis } \\
\text { to RRT }<17.6 \mathrm{hrs}\end{array}$ & $\begin{array}{l}\text { Time from AKI diagnosis to } \\
\text { RRT }>17.6 \mathrm{hrs}\end{array}$ & 6 \\
\hline $\begin{array}{l}\text { Combes } \\
2015\end{array}$ & France & RCT & $\begin{array}{l}\text { Post Cardiac } \\
\text { Surgery }\end{array}$ & $40 / 112$ & $40 / 112$ & $\begin{array}{l}\text { Early: SOFA } 11.5 \\
\text { Late: SOFA } 12.0\end{array}$ & $\begin{array}{l}\text { RRT initiated }<24 \mathrm{hrs} \text { and } \\
\text { continued } \\
\text { for min of } 48 \mathrm{hrs}\end{array}$ & Traditional indications for RRT & $\mathrm{H}$ \\
\hline $\begin{array}{l}\text { Yang } \\
2016\end{array}$ & China & Retrospective & $\begin{array}{l}\text { Post Cardiac } \\
\text { Surgery }\end{array}$ & $20 / 59$ & $80 / 154$ & $\begin{array}{l}\text { Early: APACHE2=21.4.; } \\
\text { Late: APACHE2 }=23.1\end{array}$ & $\begin{array}{l}\text { AKI in absence of } \\
\text { traditional indications } \\
\text { for RRT; persistence of } \\
\text { hypotension (for more } \\
\text { than } 6 \mathrm{~h} \text { ) despite preload } \\
\text { optimization; }\end{array}$ & Traditional indications for RRT & 7 \\
\hline \multicolumn{10}{|c|}{ Early time to RRT $<48 \mathrm{~h}$} \\
\hline $\begin{array}{l}\text { Durmaz } \\
2003\end{array}$ & Turkey & RCT & $\begin{array}{l}\text { Post Cardiac } \\
\text { Surgery }\end{array}$ & $1 / 21$ & $7 / 23$ & NR & $\begin{array}{l}\text { Cr rise }>10 \% \text { from pre-op } \\
\text { level } \\
\text { within } 48 \mathrm{hrsof} \text { surgery }\end{array}$ & $\begin{array}{l}\text { Cr rise }>50 \% \text { from pre-op level; } \\
\text { or Urine output }<400 \mathrm{ml} / 24 \mathrm{hrs}\end{array}$ & $\mathrm{L}$ \\
\hline $\begin{array}{l}\text { Lyem } \\
2009\end{array}$ & Turkey & Prospective & $\begin{array}{l}\text { Post Cardiac } \\
\text { Surgery }\end{array}$ & $5 / 95$ & $6 / 90$ & NR & $\begin{array}{l}\text { Low urine output } \\
\text { triggering RRT started } \\
<48 \mathrm{hrs} \text {; Evidence of } 50 \% \\
\text { increase in BUN, }\end{array}$ & $\begin{array}{l}\text { Time }>48 \mathrm{hrs} \text { to start of RRT for similar } \\
\text { markers of renal failure managed } \\
\text { medically for minimum } 48 \mathrm{hrs}\end{array}$ & 7 \\
\hline $\begin{array}{l}\text { Bagshaw } \\
2009\end{array}$ & $\begin{array}{l}\text { Multi } \\
\text { countries }\end{array}$ & Prospective & Multisystem & $462 / 785$ & $304 / 442$ & $\begin{array}{l}\text { Early: SOFA } 10.9 ; \\
\text { Late: SOFA } 10.7 \\
p=0.04\end{array}$ & $\begin{array}{l}\text { RRT started }<2 \mathrm{~d} \text { from } \\
\text { ICU admission }\end{array}$ & RRT started $>2 \mathrm{~d}$ from ICU admission & 7 \\
\hline $\begin{array}{l}\text { Perez } \\
2012\end{array}$ & Spain & Prospective & $\begin{array}{l}\text { Multisystem } \\
\text { Sepsis }\end{array}$ & $71 / 135$ & $78 / 109$ & $\begin{array}{l}\text { Early: SOFA } 12 \\
\text { Late: SOFA } 11\end{array}$ & $\begin{array}{l}\text { Time from ICU admission } \\
\text { to RRT }<48 \mathrm{~h}\end{array}$ & Time from ICU admission to RRT $>48 \mathrm{~h}$ & 5 \\
\hline $\begin{array}{l}\operatorname{Lim} \\
2014\end{array}$ & Singapore & Prospective & Multisystem & $37 / 56$ & $36 / 84$ & $\begin{array}{l}\text { Early: SOFA 11; } \\
\text { Late: SOFA 7; } \\
\mathrm{p}=0.001\end{array}$ & $\begin{array}{l}\text { RRT started }<2 \mathrm{~d} \text { from } \\
\text { admission; } \\
\text { Traditional indications } \\
\text { for RRT }\end{array}$ & $\begin{array}{l}\text { RRT started }>2 \mathrm{~d} \text { from admission; } \\
\text { AKIN stage } 1 \text { or } 2 \text { with indication or } \\
\text { AKIN stage } 3\end{array}$ & 6 \\
\hline $\begin{array}{l}\text { Hyung } \\
2016\end{array}$ & Korea & Retrospective & $\begin{array}{l}\text { Multisystem } \\
\text { Sepsis }\end{array}$ & $9 / 30$ & $17 / 30$ & $\begin{array}{l}\text { Early: APACHE2 }=22.9 \\
\text { Late: } \text { APACHE2 } 21.1\end{array}$ & Time to RRT $<26.4 \mathrm{~h}$ & Time to RRT $>26.4 \mathrm{~h}$ & 6 \\
\hline \multicolumn{10}{|c|}{ Early time to RRT $<72 \mathrm{~h}$} \\
\hline $\begin{array}{l}\text { Sugahara } \\
2004\end{array}$ & Japan & $\mathrm{RCT}$ & $\begin{array}{l}\text { Post Cardiac } \\
\text { Surgery }\end{array}$ & $12 / 14$ & $2 / 14$ & $\begin{array}{l}\text { Early: APACHE } 2=18 \\
\text { Late: }\end{array}$ & $\begin{array}{l}\text { Mean time to RRT start } \\
1.7 \mathrm{~d} \pm 0.8 \text { post op; UOP } \\
<20 \mathrm{ml} / \mathrm{hrs} \times 2 \mathrm{hrs}+\text { OR } \\
\text { UOP }<500 \mathrm{ml} / \text { day }\end{array}$ & $\begin{array}{l}\text { Mean time to RRT start } 18 \mathrm{~d} \pm 0.9 \text { post } \\
\text { op; UOP }<30 \mathrm{ml} / \mathrm{hrs} \times 3 \mathrm{hrs} \text { OR } \\
\text { UOP }<750 \mathrm{ml} / \mathrm{day}\end{array}$ & $\mathrm{L}$ \\
\hline $\begin{array}{l}\text { Sabater } \\
2009\end{array}$ & Spain & Prospective & Multisystem & $21 / 44$ & $68 / 104$ & $\begin{array}{l}\text { Early: } \mathrm{APACHE} 2=26 \\
\text { Late: } \mathrm{APACHE} 2=24\end{array}$ & $\begin{array}{l}\text { Mean RRT start } 2.2 \mathrm{~d} \\
\text { post ICU admit (RIFLE } \\
\text { criteria: RISK \& } \\
\text { INJURY) }\end{array}$ & $\begin{array}{l}\text { Mean RRT start } 6.4 \mathrm{~d} \text { post ICU admit } \\
\text { (RIFLE criteria: FAILURE) }\end{array}$ & 7 \\
\hline $\begin{array}{l}\text { Fernandez } \\
2011\end{array}$ & Spain & Retrospective & $\begin{array}{l}\text { Post Cardiac } \\
\text { Surgery }\end{array}$ & $59 / 111$ & $74 / 92$ & NR & $\begin{array}{l}\text { RRT started }<3 \mathrm{~d} \text { after } \\
\text { cardiac surgery }\end{array}$ & RRT started $>3 \mathrm{~d}$ after cardiac surgery & 5 \\
\hline $\begin{array}{l}\text { Shiao } \\
2012\end{array}$ & China & Retrospective & Surgical & $236 / 436$ & $143 / 212$ & $\begin{array}{l}\text { Early: SOFA } 11.4 \\
\text { Late: SOFA } 11.3\end{array}$ & $\begin{array}{l}\text { Time to development } \\
\text { of traditional RRT } \\
\text { indications }<3 \mathrm{~d} \text {; Mean } \\
\text { time to start of RRT } 1.4 \mathrm{~d}\end{array}$ & $\begin{array}{l}\text { Traditional RRT indications AND start } \\
\text { of RRT }>3 \mathrm{~d} \text {; Mean time to start of } \\
\text { RRT 18d }\end{array}$ & 6 \\
\hline \multicolumn{10}{|c|}{ Early time to RRT $>72 \mathrm{~h}$} \\
\hline $\begin{array}{l}\text { Gettings } \\
1999\end{array}$ & USA & Retrospective & $\begin{array}{l}\text { Multisystem; } \\
\text { Trauma }\end{array}$ & $25 / 41$ & $47 / 59$ & $\begin{array}{l}\text { Early ISS }=33.0 \\
\text { Late ISS }=37.2\end{array}$ & $\begin{array}{l}\text { Mean RRT start post } \\
\text { admission10d; BUN } \\
<60 \mathrm{mg} / \mathrm{dl} \text { AND Oliguria, } \\
\text { Vol overload, Electrolytes, } \\
\text { Uremia; }\end{array}$ & $\begin{array}{l}\text { Mean RRT start post admission 19d; } \\
\text { BUN }>60 \mathrm{mg} / \mathrm{dL} \text { AND Oliguria, } \\
\text { Electrolytes, Uremia; }\end{array}$ & 5 \\
\hline $\begin{array}{l}\text { Shiao } \\
2009\end{array}$ & China & Prospective & $\begin{array}{l}\text { Major } \\
\text { Abdominal } \\
\text { Surgery }\end{array}$ & $22 / 51$ & $34 / 47$ & $\begin{array}{l}\text { Early: SOFA } 8.3 \\
\text { Late: SOFA } 8.5\end{array}$ & $\begin{array}{l}\text { Mean Time to RRT from } \\
\text { ICU Admit =7.3d (RIFLE } \\
\text { criteria: } \\
\text { RISK or pre-RISK } \\
\text { criteria) }\end{array}$ & $\begin{array}{l}\text { Mean Time to RRT from ICU Admit = } \\
\text { 8.4d (RIFLE criteria: } \\
\text { INJURY or FAILURE criteria) }\end{array}$ & 7 \\
\hline $\begin{array}{l}\text { Chung } \\
2009\end{array}$ & US & Retrospective & $\begin{array}{l}\text { Severe } \\
\text { Burned } \\
\text { Patients }\end{array}$ & $9 / 29$ & $24 / 28$ & $\begin{array}{l}\text { Early: SOFA } 13 \\
\text { Late: SOFA } 13\end{array}$ & $\begin{array}{l}\text { Mean time from admit } \\
\text { to RRT }= \\
17 \text { days; AKIN } \\
\text { stage } 2(+ \text { shock }) / 3\end{array}$ & $\begin{array}{l}\text { Mean time from admit to AKIN stage } \\
2(+ \text { shock }) / 3 \text { but not dialyzed }=23 \text { days }\end{array}$ & 6 \\
\hline
\end{tabular}

(Continued) 


\begin{tabular}{|c|c|c|c|c|c|c|c|c|c|}
\hline $\begin{array}{l}\text { Auther, } \\
\text { Year }\end{array}$ & Country & Study Design & Population & $\begin{array}{c}\text { Early } \\
\text { Mortality }\end{array}$ & $\begin{array}{c}\text { Late } \\
\text { Mortality }\end{array}$ & $\begin{array}{l}\text { Severity of } \\
\text { Illness }\end{array}$ & Early RRT Criteria & Late RRT Criteria & Quality \\
\hline $\begin{array}{l}\text { Carl } \\
2010\end{array}$ & US & Retrospective & $\begin{array}{l}\text { Multisystem; } \\
\text { Sepsis }\end{array}$ & $44 / 85$ & $42 / 62$ & $\begin{array}{l}\text { Early: APACHE2 }=24.8 ; \\
\text { Late: APACHE2 }=24.7\end{array}$ & $\begin{array}{l}\text { Mean ICU stay prior to } \\
\text { RRT }=6.3 \mathrm{~d} \text {; } \\
\text { BUN }<100 \mathrm{mg} / \mathrm{dL}+\text { AKIN } \\
\text { stage }>2\end{array}$ & $\begin{array}{l}\text { Mean ICU stay prior to RRT }=12.3 \mathrm{~d} \text {; } \\
\text { BUN }>100 \mathrm{mg} / \mathrm{dL}+\text { AKIN stage }>2 \text {; }\end{array}$ & 7 \\
\hline $\begin{array}{l}\text { Hyung } \\
2012\end{array}$ & Korea & Retrospective & Multisystem & $75 / 105$ & $81 / 105$ & $\begin{array}{l}\text { Early: SOFA } 14.4 \\
\text { Late: SOFA } 14.4\end{array}$ & $\begin{array}{l}\text { Time from ICU admission } \\
\text { to } \mathrm{RRT}=4.7 \mathrm{~d}\end{array}$ & Time from ICU admission to RRT $=4.8 \mathrm{~d}$ & 7 \\
\hline \multicolumn{10}{|c|}{ RRT initiated base on biochemical indicators; Meantime to initiation of RRT not specified } \\
\hline $\begin{array}{l}\text { Kresse } \\
1999\end{array}$ & Germany & Retrospective & Multisystem & $83 / 141$ & $102 / 128$ & NR & $\begin{array}{l}\mathrm{BUN} \leq 34 \mathrm{mmol} / \mathrm{L}, \mathrm{sCr} \\
\text { 380umol/L, and urine } \\
\text { output } 924 \mathrm{ml} / 24 \mathrm{~h}\end{array}$ & $\begin{array}{l}\text { BUN }>34 \mathrm{mmol} / \mathrm{L}, \mathrm{sCr} 477 \mathrm{umol} / \mathrm{L} \text {, and } \\
\text { urine output } 525 \mathrm{ml} / 24 \mathrm{~h}\end{array}$ & 7 \\
\hline $\begin{array}{l}\text { Splendiani } \\
2001\end{array}$ & Italy & Retrospective & Multisystem & $6 / 14$ & $3 / 13$ & NR & $\mathrm{BUN} \leq 33 \mathrm{mmol} / \mathrm{L}$ & $\begin{array}{l}\text { BUN }>59 \mathrm{mmol} / \mathrm{L} \text { and } / \text { or severe } \\
\text { electrolyte disturbances }\end{array}$ & 5 \\
\hline $\begin{array}{l}\text { Tsai } \\
2005\end{array}$ & China & Retrospective & Multisystem & $42 / 67$ & $30 / 31$ & NR & $\mathrm{BUN}<29 \mathrm{mmol} / \mathrm{L}$ & $\mathrm{BUN}>29 \mathrm{mmol} / \mathrm{L}$ & 5 \\
\hline $\begin{array}{l}\text { Liu } \\
2006\end{array}$ & $\begin{array}{l}\text { Multi } \\
\text { countries }\end{array}$ & Prospective & Multisystem & $43 / 122$ & $50 / 121$ & NR & $\begin{array}{l}\text { Azotemia defined by } \\
\text { BUN }<76 \mathrm{mg} / \mathrm{dL}\end{array}$ & Azotemia defined by BUN $>76 \mathrm{mg} / \mathrm{dL}$ & 6 \\
\hline $\begin{array}{l}\text { Payen } \\
2009\end{array}$ & France & RCT & Multisystem & $20 / 37$ & $17 / 39$ & $\begin{array}{l}\text { Early: SOFA } 11.6 \\
\text { Late: SOFA } 10.4\end{array}$ & $\begin{array}{l}\text { RRT } \times 96 \text { hrs w/diagnosis } \\
\text { of 'sepsis' }\end{array}$ & $\begin{array}{l}\text { No RRT; unless metabolic renal failure } \\
\text { \& classic indications for RRT present }\end{array}$ & M \\
\hline $\begin{array}{l}\text { Elsevivrs } \\
2010\end{array}$ & Belgium & Prospective & Multisystem & $379 / 653$ & $280 / 650$ & $\begin{array}{l}\text { Early: SOFA } 9.9 ; \\
\text { Late: SOFA } 8.5 \\
p=0.001\end{array}$ & Serum $\mathrm{Cr}>2 \mathrm{mg} / \mathrm{dL}$ & No RRT & 5 \\
\hline $\begin{array}{l}\text { Konopka } \\
2011\end{array}$ & Poland & Retrospective & Multisystem & $17 / 25$ & $11 / 12$ & NR & $\begin{array}{l}\text { As soon as AKI was } \\
\text { diagnosed }\end{array}$ & $\begin{array}{l}\text { After full treatment for } \mathrm{HF} \text { and } \\
\text { unsuccessful pharmacological treatment } \\
\text { of complicating AKI }\end{array}$ & 5 \\
\hline $\begin{array}{l}\text { Chou } \\
2011\end{array}$ & China & Retrospective & $\begin{array}{l}\text { Sepsis; } \\
\text { Surgery ICU }\end{array}$ & $135 / 192$ & $124 / 178$ & $\begin{array}{l}\text { Early: SOFA } 10.8 \\
\text { Late: SOFA } 11.6\end{array}$ & $\begin{array}{l}\text { RIFLE criteria: RISK or } \\
\text { pre-RISK }\end{array}$ & RIFLE criteria: INJURY or FAILURE & 6 \\
\hline Nascimento2012 & Brazil & Retrospective & Multisystem & $9 / 23$ & $43 / 63$ & $\begin{array}{l}\text { Early: APACHE } 2=21 \\
\text { Late: APACHE } 2=28\end{array}$ & $\mathrm{BUN} \leq 26.7 \mathrm{mmol} / \mathrm{L}$ & $\mathrm{BUN}>26.7 \mathrm{mmol} / \mathrm{L}$ & 6 \\
\hline $\begin{array}{l}\text { Wu SC } \\
2012\end{array}$ & China & Retrospective & $\begin{array}{l}\text { Multisystem } \\
\text { Surgery }\end{array}$ & $10 / 20$ & $45 / 53$ & $\begin{array}{l}\text { Early: SOFA } 9.5 ; \\
\text { Late: SOFA } 10.0\end{array}$ & RIFLE criteria: RISK & RIFLE criteria: INJURY or FAILURE & 5 \\
\hline $\begin{array}{l}\mathrm{Hu} \\
2013\end{array}$ & China & Retrospective & Multisystem & $20 / / 36$ & $8 / 13$ & $\begin{array}{l}\text { Early: SOFA } 9.3 \\
\text { Late: SOFA } 11.5\end{array}$ & $\begin{array}{l}\text { AKIN 1and } 2(\mathrm{Cr} \\
>200-300 \% \text { baseline \& } \\
\text { Urine }<0.5 \mathrm{cc} / \mathrm{kg} / \mathrm{h} \text { for } \\
>12 \mathrm{~h})\end{array}$ & $\begin{array}{l}\text { AKIN } 3(\mathrm{Cr} \geq 354 \mu \mathrm{mol} / \mathrm{L} \text { or } \mathrm{Cr}>300 \% \\
\text { baseline \& urine }<0.3 \mathrm{cc} / \mathrm{kg} / \mathrm{h} \text { for } 24 \mathrm{~h} \text { or } \\
\text { anuria }>12 \mathrm{~h})\end{array}$ & 5 \\
\hline $\begin{array}{l}\text { Jamle } \\
2013\end{array}$ & India & RCT & Multisystem & $21 / 102$ & $13 / 106$ & $\begin{array}{l}\text { Early: SOFA } 7.3 ; \\
\text { Late: SOFA } 8.2\end{array}$ & $\mathrm{Cr}>618 \mu \mathrm{mol} / \mathrm{L}$ & Traditional indications for RRT & M \\
\hline $\begin{array}{l}\text { Gaudry } \\
2014\end{array}$ & France & Retrospective & $\begin{array}{l}\text { Multisystem; } \\
\text { Sepsis }\end{array}$ & $44 / 91$ & $29 / 112$ & $\begin{array}{l}\text { Early: SOFA 9; } \\
\text { Late: SOFA } 8 \\
\mathrm{P}<0.01\end{array}$ & $\begin{array}{l}\text { RRT criteria: } \mathrm{Cr} \\
\geq 300 \mu \mathrm{mol} / \mathrm{L} \text {, } \\
\text { Urea }>25 \mathrm{mmol} / \mathrm{L}, \mathrm{K} \\
>6.5 \mathrm{mmol} / \mathrm{L} \text {, } \\
\mathrm{pH}<7.2 \text {, Oliguria, Vol } \\
\text { overload, }\end{array}$ & No RRT & 5 \\
\hline $\begin{array}{l}\operatorname{Tian}\left(46^{1}\right) \\
2014\end{array}$ & China & Retrospective & $\begin{array}{l}\text { Multisystem; } \\
\text { Sepsis }\end{array}$ & $5 / 23$ & $11 / 26$ & $\begin{array}{l}\text { Early: SOFA } 7.6 \\
\text { Late: SOFA } 8.4\end{array}$ & $\begin{array}{l}\text { AKIN } 1(\mathrm{Cr} \geq 26.4 \mu \mathrm{mol} / \mathrm{L} \\
\text { or }>150-200 \% \text { baseline } \\
\& \text { urine }<0.5 \mathrm{cc} / \mathrm{kg} / \mathrm{h} \\
\text { for }>6 \mathrm{~h})\end{array}$ & No RRT & 6 \\
\hline $\begin{array}{l}\operatorname{Tian}\left(46^{2}\right) \\
2014\end{array}$ & China & Retrospective & $\begin{array}{l}\text { Multisystem; } \\
\text { Sepsis }\end{array}$ & $12 / 31$ & $14 / 21$ & $\begin{array}{l}\text { Early: SOFA } 9.3 \\
\text { Late: SOFA } 9.6\end{array}$ & $\begin{array}{l}\text { AKIN } 2(\mathrm{Cr}>200-300 \% \\
\text { baseline } \& \\
\text { Urine }<0.5 \mathrm{cc} / \mathrm{kg} / \mathrm{h} \text { for } \\
>12 \mathrm{~h})\end{array}$ & No RRT & 6 \\
\hline $\begin{array}{l}\operatorname{Tian}\left(46^{3}\right) \\
2014\end{array}$ & China & Retrospective & $\begin{array}{l}\text { Multisystem; } \\
\text { Sepsis }\end{array}$ & $31 / 46$ & $11 / 13$ & $\begin{array}{l}\text { Early: SOFA 10; } \\
\text { Late: SOFA } 11.2\end{array}$ & $\begin{array}{l}\text { AKIN } 3(\mathrm{Cr} \geq 354 \mu \mathrm{mol} / \mathrm{L} \\
\text { or } \mathrm{Cr}>300 \% \text { baseline } \& \\
\text { urine }<0.3 \mathrm{cc} / \mathrm{kg} / \mathrm{h} \text { for } 24 \mathrm{~h} \\
\text { or anuria }>12 \mathrm{~h})\end{array}$ & No RRT & 6 \\
\hline
\end{tabular}

LEGEN: AKI Acute kidney injury, RRT renal replacement therapy, Cr Creatinine, UOP Urine output, ICU Intensive Care Unit, AKIN Acute Kidney Injury Network, RIFLE Risk, Injury, Failure, Loss and End-stage, KDIGO Kidney Disease: Improving Global Outcomes, RCTs randomized clinical trials, Quality Score: The Cochrane Collaboration Risk of Bias tool for RCTs and Newcastle-Ottawa Scale for observational studies, H High quality: low risk of bias, M Medium quality: unclear risk of bias, L Low quality: high risk of bias, APACHE Acute Physiology and Chronic Health Evaluation, SOFA Sequential Organ Failure Assessment, NR Not reported. 
and late RRT with the study (OR, 1.07; 95\% CI, 0.79 $1.45 ; I^{2}, 73.0 \%$ ) or without the study (OR, $1.02 ; 95 \% \mathrm{CI}$, $\left.0.74-1.40 ; I^{2}, 66.8 \%\right)$. Elsevivrs et al. was a large sample trial with 1303 patients when compared to other articles including not more than 619 subjects (Figure 4).

With the meta-regression, we did not find a correlation between patient mortality and study design (RCT vs. observational), RRT modality (CRRT, IHD vs. Mixed), study quality score, severity of illness [Sequential
Organ Failure Assessment (SOFA) score], ICU admission type (surgical vs. mixed medical admissions). However, we find a correlation between patient mortality and sample size $(\mathrm{n} \geq 100$ vs. $\mathrm{n}<100, P=0.001)$ in critically ill patients with AKI.

\section{Publication bias}

No potential publication bias was observed in non-critically ill patients with AKI $(P=0.347$ for the

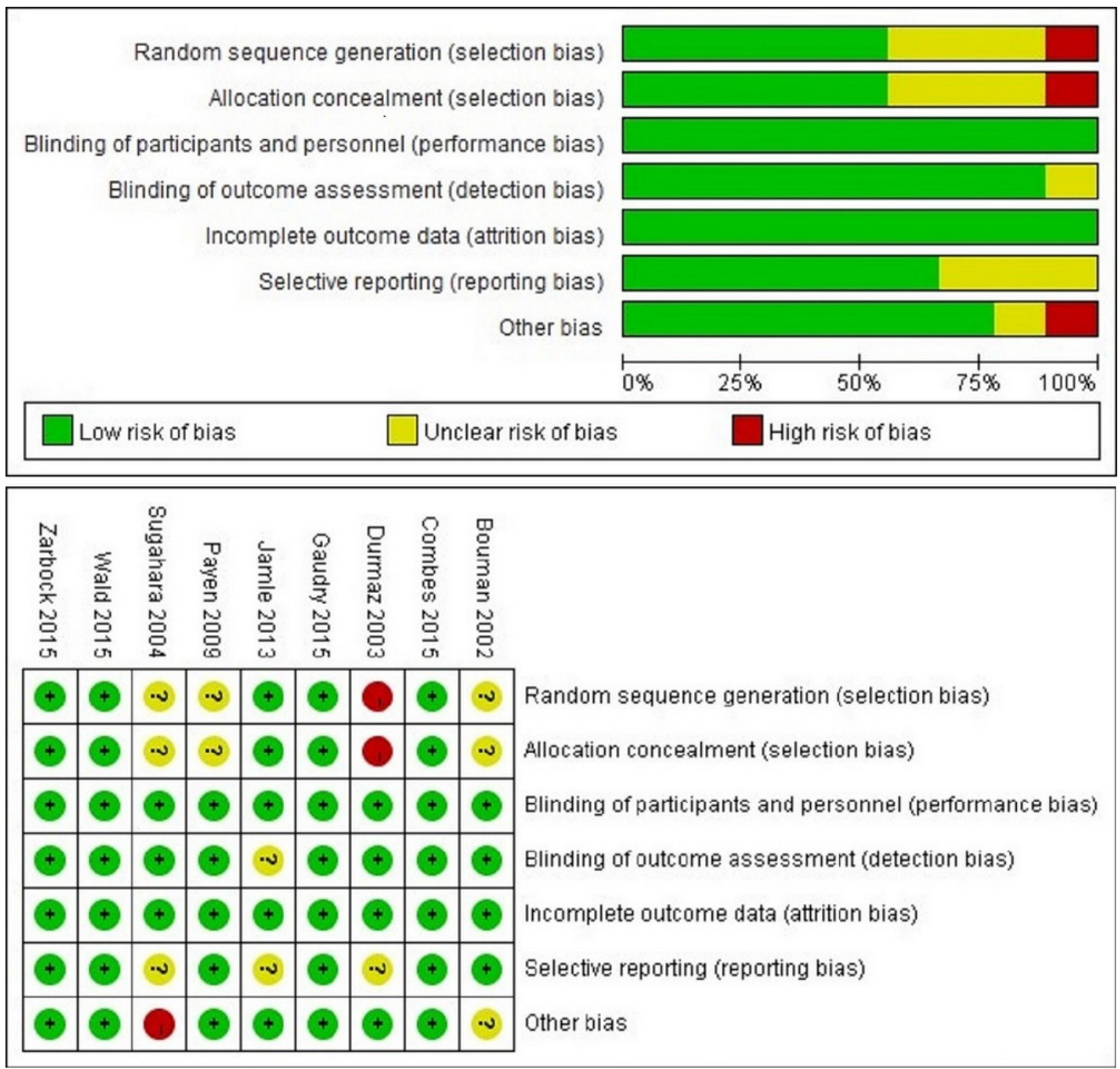

Figure 2: Risk of bias summary of early versus late RRT initiation on mortality in patients with AKI on randomized controlled trial. 
Table 2: Outcomes measures of early versus late RRT initiation

\begin{tabular}{|c|c|c|c|c|c|c|c|c|c|c|}
\hline \multirow[b]{2}{*}{$\begin{array}{l}\text { Outcome or } \\
\text { Subgroup }\end{array}$} & \multicolumn{5}{|c|}{ Group A: critically ill patients with AKI } & \multicolumn{5}{|c|}{ Group B: non-critically ill patients with AKI } \\
\hline & Studies & $\begin{array}{c}\text { No. of } \\
\text { Patients }\end{array}$ & Study Reference No & $\begin{array}{c}\text { Effect } \\
\text { Estimate } \\
(95 \% \text { CI })\end{array}$ & $p$ & Studies & $\begin{array}{l}\text { No. of } \\
\text { Patients }\end{array}$ & Study Reference No & $\begin{array}{c}\text { Effect } \\
\text { Estimate } \\
(95 \% \text { CI })\end{array}$ & $p$ \\
\hline \multicolumn{11}{|c|}{ Primary Outcomes: early versus late RRT initiation on mortality } \\
\hline All studies & 31 & 5408 & $\begin{array}{l}7-9,12,18,28-30,32,34,35,38- \\
41,43,44,46^{2}, 46^{3}, 47,48,50-59\end{array}$ & $\begin{array}{c}\text { OR, } 0.40 \\
(0.32 \text { to } 0.48)\end{array}$ & 0.001 & 20 & 4290 & $\begin{array}{l}10,11,13-17,19-23,31 \\
33,36,37,42,45,46^{1}, 49\end{array}$ & $\begin{array}{c}\text { OR, } 1.07 \\
(0.79 \text { to } 1.45)\end{array}$ & 0.000 \\
\hline \multicolumn{11}{|c|}{ Subgroup stratified by the definition of early according to time criteria and biochemical indicators on mortality } \\
\hline $\begin{array}{l}\text { Time: Early RRT } \\
<12 \mathrm{~h}\end{array}$ & 7 & 639 & $9,12,28-30,32,56$ & $\begin{array}{c}\text { OR, } 0.28 \\
(0.16 \text { to } 0.49)\end{array}$ & 0.093 & 5 & 1003 & $10,13,21,31,42$ & $\begin{array}{c}\text { OR, } 0.86 \\
(0.58 \text { to } 1.29)\end{array}$ & 0.201 \\
\hline $\begin{array}{l}\text { Time: Early RRT } \\
<24 \mathrm{~h}\end{array}$ & 4 & 534 & $34,35,53,54$ & $\begin{array}{c}\text { OR, } 0.37 \\
(0.25 \text { to } 0.54)\end{array}$ & 0.691 & 4 & 782 & $11,22,33,36$ & $\begin{array}{c}\text { OR, } 0.72 \\
(0.43 \text { to } 1.19)\end{array}$ & 0.097 \\
\hline $\begin{array}{l}\text { Time: Early RRT } \\
<48 \mathrm{~h}\end{array}$ & 3 & 1531 & $7,55,57$ & $\begin{array}{c}\text { OR, } 0.55 \\
(0.39 \text { to } 0.77)\end{array}$ & 0.236 & 3 & 368 & $17,19,37$ & $\begin{array}{c}\text { OR, } 0.82 \\
(0.18 \text { to } 3.79)\end{array}$ & 0.012 \\
\hline $\begin{array}{l}\text { Time: Early RRT } \\
<72 \mathrm{~h}\end{array}$ & 3 & 999 & $18,38,58$ & $\begin{array}{c}\text { OR, } 0.45 \\
(0.29 \text { to } 0.69)\end{array}$ & 0.145 & 1 & 28 & 16 & $\begin{array}{c}\text { OR, } 36.0 \\
(4.33 \text { to } \\
299.02)\end{array}$ & NE \\
\hline $\begin{array}{l}\text { Time: Early RRT } \\
>72 \mathrm{~h}\end{array}$ & 4 & 465 & $8,39,40,52$ & $\begin{array}{c}\text { OR, } 0.32 \\
(0.14 \text { to } 0.74)\end{array}$ & 0.015 & 0 & $\mathrm{NE}$ & $\mathrm{NE}$ & $\mathrm{NE}$ & $\mathrm{NE}$ \\
\hline $\begin{array}{l}\text { Biochemicl } \\
\text { indicators }\end{array}$ & 10 & 1240 & $41,43,44,46^{2}, 46^{3}-48,50,51,59$ & $\begin{array}{c}\text { OR, } 0.40 \\
(0.25 \text { to } 0.64)\end{array}$ & 0.009 & 7 & 2109 & $14,15,20,23,45,46^{1}, 49$ & $\begin{array}{c}\text { OR, } 1.46 \\
(0.96 \text { to } 2.23)\end{array}$ & 0.008 \\
\hline \multicolumn{11}{|c|}{ Subgroup stratified by surgical versus mixed medical admissions on mortality } \\
\hline Surgical & 9 & 1506 & $8,9,18,30,32,34,38,44,54$ & $\begin{array}{c}\mathrm{OR}, 0.33 \\
(0.22 \text { to } 0.48)\end{array}$ & 0.053 & 6 & 602 & $16,17,19,22,31,33$ & $\begin{array}{c}\text { OR, } 0.71 \\
(0.24 \text { to } 2.07)\end{array}$ & 0.000 \\
\hline Mixed medical & 22 & 3902 & $\begin{array}{l}7,12,28,29,35,39,41,43 \\
46^{2}, 46^{3}-48,50-53,55-59\end{array}$ & $\begin{array}{c}\text { OR, } 0.43 \\
(0.34 \text { to } 0.54)\end{array}$ & 0.004 & 14 & 3688 & $\begin{array}{c}10,11,13-15,20,21,23 \\
36,37,42,45,46^{1}, 49\end{array}$ & $\begin{array}{c}\text { OR, } 1.22 \\
(0.91 \text { to } 1.63)\end{array}$ & 0.000 \\
\hline \multicolumn{11}{|c|}{ Subgroup stratified by RRT modality on mortality } \\
\hline Mixed & 14 & 3442 & $\begin{array}{c}7,9,12,28,29,35,38,41 \\
43,48,53,54,55,57\end{array}$ & $\begin{array}{c}\text { OR, } 0.45 \\
(0.35 \text { to } 0.57)\end{array}$ & 0.009 & 6 & 2495 & $13,14,20,21,45,49$ & $\begin{array}{c}\text { OR, } 1.32 \\
(0.86 \text { to } 2.03)\end{array}$ & 0.000 \\
\hline CRRT & 14 & 1771 & $\begin{array}{c}8,18,32,34,39,40,44,46^{2}, \\
46^{3}, 47,50,52,55,58\end{array}$ & $\begin{array}{c}\text { OR, } 0.40 \\
(0.30 \text { to } 0.54)\end{array}$ & 0.152 & 12 & 1544 & $\begin{array}{c}10,11,15-17,22,31 \\
33,36,37,42,46^{1}\end{array}$ & $\begin{array}{c}\text { OR, } 0.92 \\
(0.58 \text { to } 1.46)\end{array}$ & 0.017 \\
\hline IHD & 3 & 255 & $30,51,59$ & $\begin{array}{c}\text { OR, } 0.11 \\
(0.03 \text { to } 0.43)\end{array}$ & 0.098 & 2 & 251 & 19,23 & $\begin{array}{c}\text { OR, } 0.56 \\
(0.04 \text { to } 8.73)\end{array}$ & 0.000 \\
\hline \multicolumn{11}{|c|}{ Secondary outcomes: ICU and Hospital LOS } \\
\hline ICU LOS & 8 & 862 & $28,34,35,38,41,46^{2}, 46^{3}, 53$ & $\begin{array}{c}\text { MD, }-0.41 \\
(-0.55 \text { to }-0.27)\end{array}$ & 0.000 & 4 & 336 & $17,19,31,46^{1}$ & $\begin{array}{c}\mathrm{MD},-1.47 \\
(-1.71 \text { to }-1.22)\end{array}$ & 0.000 \\
\hline Hospital LOS & 6 & 755 & $8,28,34,38,39,54$ & $\begin{array}{c}\text { MD, }-0.36 \\
(-0.51 \text { to }-0.21)\end{array}$ & 0.000 & 3 & 287 & $17,19,31$ & $\begin{array}{c}\mathrm{MD},-1.07 \\
(-1.31 \text { to }-0.82)\end{array}$ & 0.415 \\
\hline
\end{tabular}

LEGEN: OR odds ratio, 95\% CI confidence interval, $P$ Test for Heterogeneity, MD mean difference, RRT renal replacement therapy, ICU Intensive Care Unit, CRRT continuous renal replacement therapy, IHD intermittent hemodialysis, Mixed CRRT and/or IHD and/or other RRT modality, LOS length of stay, NE not evaluable.

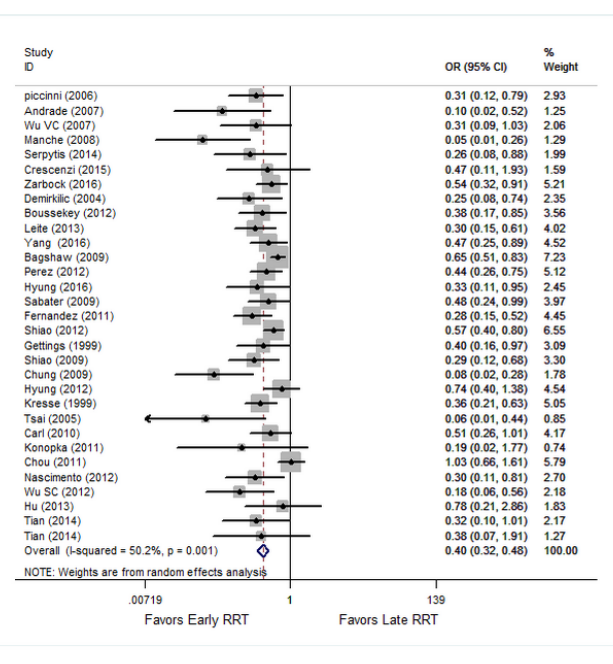

A

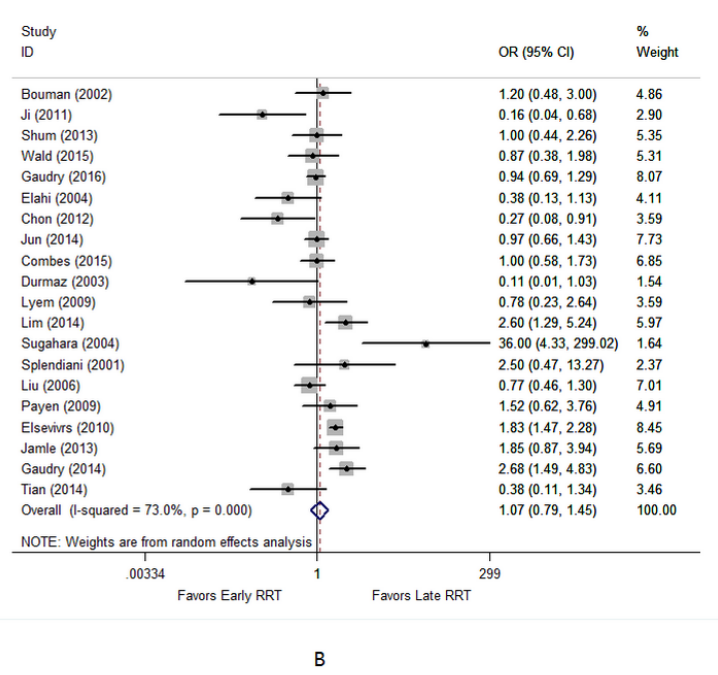

Figure 3: Forest plot shows the effect of early versus late RRT on mortality in critically ill (A) and non-critically ill patients with AKI (B). 
Begg test, and $P=0.169$ for the Egger test). Publication bias was seen in critically ill patients with AKI $(P=$ 0.001 for the Begg test, and $P=0.000$ for the Egger test, Figure 5).

\section{DISCUSSION}

We identified 49 studies reported data on the timing of RRT initiation among 9698 patients with AKI, and we found that early RRT significantly reduced the mortality compared to late RRT in critically ill patients with AKI. In addition, no significant survival benefits associated with early RRT were seen in non-critically ill patients with AKI. Early RRT was markedly associated with shortened ICU and hospital LOS compared to late RRT in both critically ill and non-critically ill patients with AKI.

Regardless of the definition of early RRT (according to time criteria or biochemical indicators), ICU admission type (surgical vs. mixed) or RRT modality (CRRT, IHD vs. Mixed), subgroup analyses of critically ill patients with AKI did reveal survival benefits from early RRT. Furthermore, subgroup analyses of non-critically ill patients with AKI showed that no evidence of survival advantage in early RRT.

In the present study, we firstly performed the meta-analysis according to the severity of illness and definition of early RRT based on time-based cutoffs for patients with AKI to investigate the time of RRT initiation. We accepted a broad definition of "critically ill patients with AKI" based on AKI with multiple-organ dysfunction syndrome [60], septic shock [40], RIFLE criteria (failure, loss of function, and end-stage kidney disease) [37, 43, 44], AKIN stages 3 [41, 42, 46] or Kidney Disease: Improving Global Outcomes (KDIGO) stage $3[12,61]$.

By the meta-regression, we found sample size was one of the sources of heterogeneity. In contrast to previous meta-analyses, we found a lower heterogeneity among studies on this topic, especially in the subgroup.

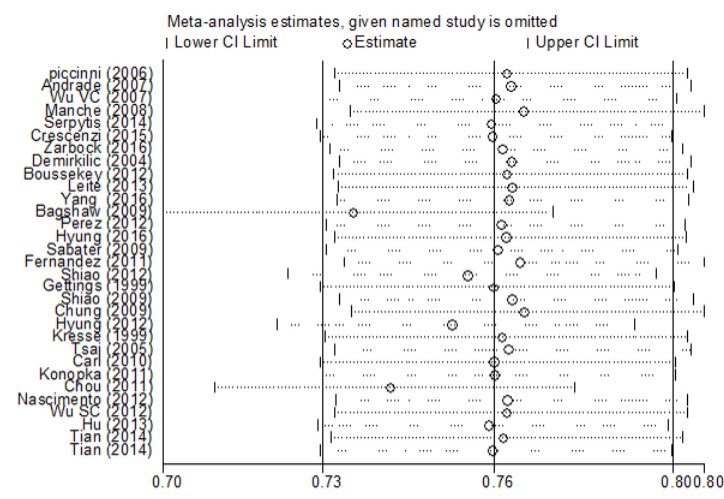

A

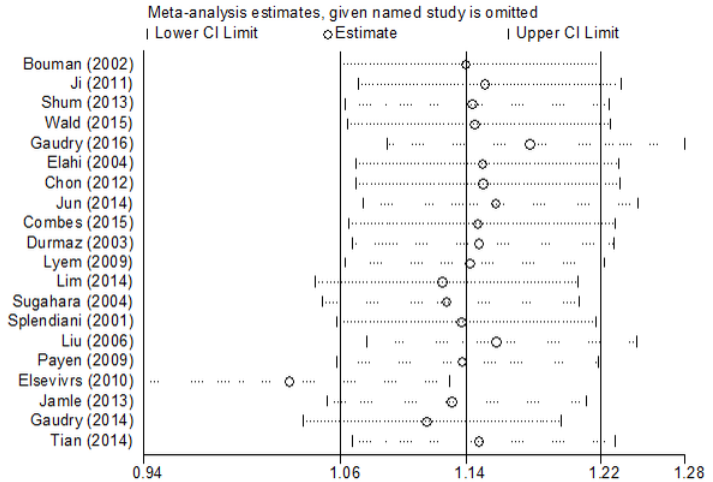

B

Figure 4: Sensitivity analyses of early versus late RRT on mortality in critically ill (A) and non-critically ill patients with AKI (B).

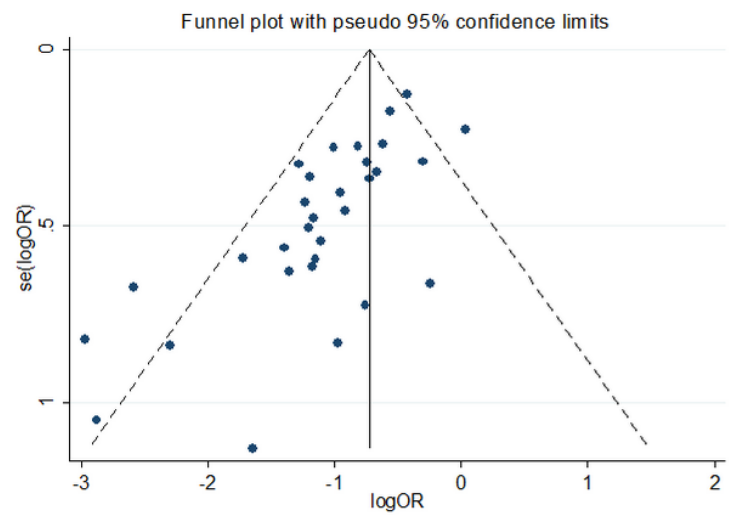

A

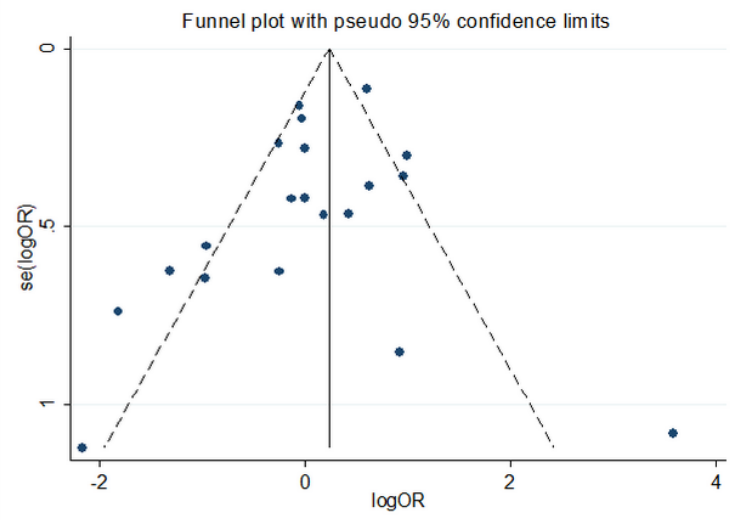

B

Figure 5: Begg's funnel plots of early versus late RRT on mortality in critically ill (A) and non-critically ill patients with AKI (B). 
We noted those critically ill patients in early RRT within 12 hours $\left(I^{2}, 44.8 \%\right), 24$ hours $\left(I^{2}, 0.0 \%\right), 48$ hours $\left(I^{2}\right.$, $30.8 \%)$, and 72 hours $\left(I^{2}, 48.2 \%\right)$ showed the lower heterogeneities, indicating that the heterogeneity may be partially explained by the definition of early RRT timing. However, we could not account for the observed heterogeneity by meta-regression according to study design, RRT modality, the study quality score, severity of the illness, and ICU admission type. Thereby, the heterogeneity observed is most likely explained by the differences in definitions for early RRT timing, the inability to account for heterogeneity in clinical practice and critical care patterns, many confounding factors that affect the mortality, publication bias, sample size and the inclusion of retrospective, prospective and RCTs.

The present systematic review has some limitations. Firstly, definitions for AKI are to some extent different in the included studies. Secondly, the definition of early RRT based on various arbitrary cutoffs for time, which ultimately downgraded the strength of evidence. Thirdly, there were publication bias and significant heterogeneity in the present study. Many confounding factors affect the mortality, and metaregression may not be enough to verify this issue. Lastly, the association with mortality is largely dependent on observational studies and might have been affected by allocation or selection bias. Thus, further high-quality RCTs focused on mortality according to the optimal time for starting RRT are necessary to fully understand the effects of early RRT for patients with AKI.

\section{MATERIALS AND METHODS}

\section{Participants, interventions and outcome measures}

We included studies that evaluated the timing of initiation of RRT in patients with AKI. For the review, early and late RRT were defined based on criteria used by the authors in their studies. early and late RRT were defined as extended time-based cutoffs (arbitrary cutoffs for time from ICU admission or development of a biochemical "start time"), or biochemical indicators [serum creatinine, serum urea, RIFLE (risk, injury, failure, loss of function, and end-stage kidney disease) classifications, Acute Kidney Injury Network (AKIN) stages, urine output, and fluid balance]. Late RRT criteria also included conventional RRT indications (hyperkalemia, acidosis or fluid overload) and expectant care (no RRT initiated). The primary outcome was mortality, and the secondary outcomes were ICU and hospital LOS.

\section{Searching strategies}

We searched the Cochrane Library, EMBASE, Global Health, MEDLINE, PubMed, the International
Clinical Trials Registry Platform, and Web of Science from January 1985 to November 2016. Owing to a low likelihood of relevance to modern RRT and critical care practices, studies published before 1985 were excluded in the present study. Keywords include acute renal failure/ acute kidney injury/renal insufficiency, mortality, renal replacement therapy/renal dialysis/ hemodialysis/dialysis. The related research references were also reviewed.

\section{Inclusion and exclusion criteria}

The inclusion criteria were as follows: (1) randomized clinical trials (RCTs) and/or observational cohort studies; (2) studies evaluating the timing of initiation of RRT in patients with AKI with direct effect on mortality; (3) complete data available to calculate odds ratio $(\mathrm{OR})$ or mean difference $(\mathrm{MD})$ with $95 \%$ confidence interval (CI); (4) clear definitions of AKI stated. Exclusion criteria were as follows: (1) data from the studies could not be extracted and analyzed; (2) duplicate publications; (3) non-human experimental studies.

\section{Study selection and data extraction}

Two investigators (Kaiping Luo and Shufang $\mathrm{Fu}$ ) independently performed the study selection. All the disagreements were resolved by discussion. Data extraction included first author, year of publication, country, study design, sample size, age, sex, RRT modality, mortality, ICU LOS, hospital LOS, and definitions of early and late RRT.

Dr. Gaudry and colleagues [13] showed that the mortality was lower in the patients who never received RRT than those received RRT early or late. Patients who received RRT late were the most severely ill at baseline, and patients who never received it were less ill at baseline. More than 50\% mortality in critically ill patients with AKI received RRT was confirmed by many randomized controlled trials [1, 3, 4, 60]]. Thus, we hypothesized that critically ill patients with AKI who receive early RRT may decrease mortality, non-critically ill patients with AKI may confer survival benefits without early RRT. Subjects were identified as being of "critically ill patients" if the late RRT group with high mortality rates $(\geq 50 \%)$, or "non-critically ill patients" if the late RRT group with low mortality rates $(<50 \%)$.

\section{Quality assessment}

The Cochrane Collaboration Risk of Bias tool was used to assess RCTs [62]. This tool consists of 6 domains and assesses 5 specific biases. A judgment of low risk, unclear risk, or high risk was provided for each domain. The Newcastle-Ottawa Scale (NOS) was used in the assessment of quality of cohort studies [63]. NOS quality assessment scale ranges from 0 to 9 stars. The star evaluates 3 main categories: selection, comparability, and outcome. 


\section{Statistical analysis}

Statistical analysis was performed using Review Manager (version 5.3) and STATA statistical software (version 12.0). We calculated OR with $95 \%$ CI for dichotomous data and MD with $95 \%$ CI for continuous data. Statistical heterogeneity of the data was quantified using the $I^{2}$ test, and the $I^{2}>50 \%$ indicated significant statistical heterogeneity. Sensitivity analysis, metaregression analyses and subgroup analysis were conducted to investigate the potential sources of heterogeneity. Publication bias was assessed by constructing a funnel plot and using the Egger regression test and the Begg rank correlation test. A $P$ value less than 0.05 was considered statistically significant.

\section{CONCLUSIONS}

Our data suggest that early RRT probably reduce the mortality, ICU and hospital LOS in critically ill patients with AKI. Inversely, early RRT in non-critically ill patients with AKI did not decrease the mortality, but shorted the ICU and hospital LOS.

\section{Abbreviations}

renal replacement therapy (RRT); acute kidney injury (AKI); odds ratio (OR); confidential intervals (CI); intensive care units (ICU); length of stay (LOS); risk, injury, failure, loss of function, and end-stage kidney disease (RIFLE); Acute Kidney Injury Network (AKIN); randomized clinical trials (RCTs); mean difference (MD); Newcastle-Ottawa Scale (NOS); continuous renal replacement therapy (CRRT); intermittent hemodialysis (IHD); Sequential Organ Failure Assessment (SOFA); Kidney Disease: Improving Global Outcomes (KDIGO).

\section{CONFLICTS OF INTEREST}

The authors have no competing interests to declare.

\section{REFERENCES}

1. Palevsky PM, Zhang JH, O’Connor TZ, Chertow GM, Crowley ST, Choudhury D, Finkel K, Kellum JA, Paganini E, Schein RM, Smith MW, Swanson KM, Thompson BT, et al, and VA/NIH Acute Renal Failure Trial Network. Intensity of renal support in critically ill patients with acute kidney injury. N Engl J Med. 2008; 359:7-20.

2. Chertow GM, Burdick E, Honour M, Bonventre JV, Bates DW. Acute kidney injury, mortality, length of stay, and costs in hospitalized patients. J Am Soc Nephrol. 2005; 16:3365-70.

3. Bellomo R, Cass A, Cole L, Finfer S, Gallagher M, Lo S, McArthur C, McGuinness S, Myburgh J, Norton R, Scheinkestel C, Su S, and RENAL Replacement Therapy
Study Investigators. Intensity of continuous renalreplacement therapy in critically ill patients. N Engl J Med. 2009; 361:1627-38.

4. Schiffl H, Lang SM, Fischer R. Daily hemodialysis and the outcome of acute renal failure. N Engl J Med. 2002; 346:305-10.

5. Ronco C, Ricci Z, De Backer D, Kellum JA, Taccone FS, Joannidis M, Pickkers P, Cantaluppi V, Turani F, Saudan P, Bellomo R, Joannes-Boyau O, Antonelli M, et al. Renal replacement therapy in acute kidney injury: controversy and consensus. Crit Care. 2015; 19:146.

6. James M, Bouchard J, Ho J, Klarenbach S, LaFrance JP, Rigatto C, Wald R, Zappitelli M, Pannu N. Canadian Society of Nephrology commentary on the 2012 KDIGO clinical practice guideline for acute kidney injury. Am $\mathrm{J}$ Kidney Dis. 2013; 61:673-85.

7. Bagshaw SM, Uchino S, Bellomo R, Morimatsu H, Morgera S, Schetz M, Tan I, Bouman C, Macedo E, Gibney N, Tolwani A, Oudemans-van Straaten HM, Ronco C, Kellum JA, and Beginning and Ending Supportive Therapy for the Kidney (BEST Kidney) Investigators. Timing of renal replacement therapy and clinical outcomes in critically ill patients with severe acute kidney injury. J Crit Care. 2009; 24:129-40.

8. Shiao CC, Wu VC, Li WY, Lin YF, Hu FC, Young GH, Kuo CC, Kao TW, Huang DM, Chen YM, Tsai PR, Lin SL, Chou NK, et al, and National Taiwan University Surgical Intensive Care Unit-Associated Renal Failure Study Group. Late initiation of renal replacement therapy is associated with worse outcomes in acute kidney injury after major abdominal surgery. Crit Care. 2009; 13:R171.

9. Wu VC, Ko WJ, Chang HW, Chen YS, Chen YW, Chen YM, Hu FC, Lin YH, Tsai PR, Wu KD. Early renal replacement therapy in patients with postoperative acute liver failure associated with acute renal failure: effect on postoperative outcomes. J Am Coll Surg. 2007; 205:266-76.

10. Bouman CS, Oudemans-Van Straaten HM, Tijssen JG, Zandstra DF, Kesecioglu J. Effects of early high-volume continuous venovenous hemofiltration on survival and recovery of renal function in intensive care patients with acute renal failure: a prospective, randomized trial. Crit Care Med. 2002; 30:2205-11.

11. Jun M, Bellomo R, Cass A, Gallagher M, Lo S, Lee J, and Randomized Evaluation of Normal Versus Augmented Level of Replacement Therapy (RENAL) Study Investigators. Timing of renal replacement therapy and patient outcomes in the randomized evaluation of normal versus augmented level of replacement therapy study. Crit Care Med. 2014; 42:1756-65.

12. Zarbock A, Kellum JA, Schmidt C, Van Aken H, Wempe C, Pavenstädt H, Boanta A, Gerß J, Meersch M. Effect of Early vs Delayed Initiation of Renal Replacement Therapy on Mortality in Critically Ill Patients With Acute Kidney Injury: The ELAIN Randomized Clinical Trial. JAMA. 2016; 315:2190-99. 
13. Gaudry S, Hajage D, Schortgen F, Martin-Lefevre L, Pons B, Boulet E, Boyer A, Chevrel G, Lerolle N, Carpentier D, de Prost N, Lautrette A, Bretagnol A, et al, and AKIKI Study Group. Initiation Strategies for Renal-Replacement Therapy in the Intensive Care Unit. N Engl J Med. 2016; 375:122-33.

14. Liu KD, Himmelfarb J, Paganini E, Ikizler TA, Soroko SH, Mehta RL, Chertow GM. Timing of initiation of dialysis in critically ill patients with acute kidney injury. Clin J Am Soc Nephrol. 2006; 1:915-19.

15. Payen D, Mateo J, Cavaillon JM, Fraisse F, Floriot C, Vicaut E, and Hemofiltration and Sepsis Group of the Collège National de Réanimation et de Médecine d'Urgence des Hôpitaux extra-Universitaires. Impact of continuous venovenous hemofiltration on organ failure during the early phase of severe sepsis: a randomized controlled trial. Crit Care Med. 2009; 37:803-10.

16. Sugahara S, Suzuki H. Early start on continuous hemodialysis therapy improves survival rate in patients with acute renal failure following coronary bypass surgery. Hemodial Int. 2004; 8:320-25.

17. Iyem H, Tavli M, Akcicek F, Büket S. Importance of early dialysis for acute renal failure after an open-heart surgery. Hemodial Int. 2009; 13:55-61.

18. Shiao CC, Ko WJ, Wu VC, Huang TM, Lai CF, Lin YF, Chao CT, Chu TS, Tsai HB, Wu PC, Young GH, Kao TW, Huang JW, et al, and National Taiwan University Hospital Study Group on Acute Renal Failure (NSARF). U-curve association between timing of renal replacement therapy initiation and in-hospital mortality in postoperative acute kidney injury. PLoS One. 2012; 7:e42952.

19. Durmaz I, Yagdi T, Calkavur T, Mahmudov R, Apaydin AZ, Posacioglu H, Atay Y, Engin C. Prophylactic dialysis in patients with renal dysfunction undergoing on-pump coronary artery bypass surgery. Ann Thorac Surg. 2003; 75:859-64.

20. Elseviers MM, Lins RL, Van der Niepen P, Hoste E, Malbrain ML, Damas P, Devriendt J, and SHARF investigators. Renal replacement therapy is an independent risk factor for mortality in critically ill patients with acute kidney injury. Crit Care. 2010; 14:R221.

21. Wald R, Adhikari NK, Smith OM, Weir MA, Pope K, Cohen A, Thorpe K, McIntyre L, Lamontagne F, Soth M, Herridge M, Lapinsky S, Clark E, et al, and Canadian Critical Care Trials Group. Comparison of standard and accelerated initiation of renal replacement therapy in acute kidney injury. Kidney Int. 2015; 88:897-904.

22. Combes A, Bréchot N, Amour J, Cozic N, Lebreton G, Guidon C, Zogheib E, Thiranos JC, Rigal JC, Bastien O, Benhaoua H, Abry B, Ouattara A, et al. Early High-Volume Hemofiltration versus Standard Care for Post-Cardiac Surgery Shock. The HEROICS Study. Am J Respir Crit Care Med. 2015; 192:1179-90.

23. Jamale TE, Hase NK, Kulkarni M, Pradeep KJ, Keskar V, Jawale S, Mahajan D. Earlier-start versus usual-start dialysis in patients with community-acquired acute kidney injury: a randomized controlled trial. Am J Kidney Dis. 2013; 62:1116-21.

24. Seabra VF, Balk EM, Liangos O, Sosa MA, Cendoroglo M, Jaber BL. Timing of renal replacement therapy initiation in acute renal failure: a meta-analysis. Am J Kidney Dis. 2008; 52:272-84.

25. Karvellas CJ, Farhat MR, Sajjad I, Mogensen SS, Leung AA, Wald R, Bagshaw SM. A comparison of early versus late initiation of renal replacement therapy in critically ill patients with acute kidney injury: a systematic review and meta-analysis. Crit Care. 2011; 15:R72.

26. Wang C, Lv LS, Huang H, Guan J, Ye Z, Li S, Wang Y, Lou $\mathrm{T}$, Liu X. Initiation time of renal replacement therapy on patients with acute kidney injury: A systematic review and meta-analysis of 8179 participants. Nephrology (Carlton). 2017; 22:7-18.

27. Wierstra BT, Kadri S, Alomar S, Burbano X, Barrisford GW, Kao RL. The impact of "early" versus "late" initiation of renal replacement therapy in critical care patients with acute kidney injury: a systematic review and evidence synthesis. Crit Care. 2016; 20:122.

28. Piccinni P, Dan M, Barbacini S, Carraro R, Lieta E, Marafon S, Zamperetti N, Brendolan A, D'Intini V, Tetta C, Bellomo R, Ronco C. Early isovolaemic haemofiltration in oliguric patients with septic shock. Intensive Care Med. 2006; 32:80-86.

29. Andrade L, Cleto S, Seguro AC. Door-to-dialysis time and daily hemodialysis in patients with leptospirosis: impact on mortality. Clin J Am Soc Nephrol. 2007; 2:739-44.

30. Manché A, Casha A, Rychter J, Farrugia E, Debono M. Early dialysis in acute kidney injury after cardiac surgery. Interact Cardiovasc Thorac Surg. 2008; 7:829-32.

31. Ji Q, Mei Y, Wang X, Feng J, Cai J, Zhou Y, Sun Y, Xie $\mathrm{S}, \mathrm{Hu} \mathrm{D}$. Timing of continuous veno-venous hemodialysis in the treatment of acute renal failure following cardiac surgery. Heart Vessels. 2011; 26:183-89.

32. Crescenzi G, Torracca L, Pierri MD, Rosica C, Munch C, Capestro F. 'Early' and 'late' timing for renal replacement therapy in acute kidney injury after cardiac surgery: a prospective, interventional, controlled, single-centre trial. Interact Cardiovasc Thorac Surg. 2015; 20:616-21.

33. Elahi MM, Lim MY, Joseph RN, Dhannapuneni RR, Spyt TJ. Early hemofiltration improves survival in post-cardiotomy patients with acute renal failure. Eur $\mathrm{J}$ Cardiothorac Surg. 2004; 26:1027-31.

34. Demirkiliç U, Kuralay E, Yenicesu M, Cağlar K, Oz BS, Cingöz F, Günay C, Yildirim V, Ceylan S, Arslan M, Vural A, Tatar H. Timing of replacement therapy for acute renal failure after cardiac surgery. J Card Surg. 2004; 19:17-20.

35. Boussekey N, Capron B, Delannoy PY, Devos P, Alfandari S, Chiche A, Meybeck A, Georges H, Leroy O. Survival in critically ill patients with acute kidney injury treated with early hemodiafiltration. Int J Artif Organs. 2012; 35:1039-46. 
36. Chon GR, Chang JW, Huh JW, Lim CM, Koh Y, Park SK, Park JS, Hong SB. A comparison of the time from sepsis to inception of continuous renal replacement therapy versus RIFLE criteria in patients with septic acute kidney injury. Shock. 2012; 38:30-36.

37. Lim CC, Tan CS, Kaushik M, Tan HK. Initiating acute dialysis at earlier Acute Kidney Injury Network stage in critically ill patients without traditional indications does not improve outcome: a prospective cohort study. Nephrology (Carlton). 2015; 20:148-54.

38. García-Fernández N, Pérez-Valdivieso JR, Bes-Rastrollo M, Vives M, Lavilla J, Herreros J, Monedero P, and GEDRCC. Timing of renal replacement therapy after cardiac surgery: a retrospective multicenter Spanish cohort study. Blood Purif. 2011; 32:104-11.

39. Gettings LG, Reynolds HN, Scalea T. Outcome in posttraumatic acute renal failure when continuous renal replacement therapy is applied early vs. late. Intensive Care Med. 1999; 25:805-13.

40. Chung KK, Lundy JB, Matson JR, Renz EM, White CE, King BT, Barillo DJ, Jones JA, Cancio LC, Blackbourne LH, Wolf SE. Continuous venovenous hemofiltration in severely burned patients with acute kidney injury: a cohort study. Crit Care. 2009; 13:R62.

41. Carl DE, Grossman C, Behnke M, Sessler CN, Gehr TW. Effect of timing of dialysis on mortality in critically ill, septic patients with acute renal failure. Hemodial Int. 2010; 14:11-17.

42. Shum HP, Chan KC, Kwan MC, Yeung AW, Cheung EW, Yan WW. Timing for initiation of continuous renal replacement therapy in patients with septic shock and acute kidney injury. Ther Apher Dial. 2013; 17:305-10.

43. Chou YH, Huang TM, Wu VC, Wang CY, Shiao CC, Lai CF, Tsai HB, Chao CT, Young GH, Wang WJ, Kao TW, Lin SL, Han YY, et al, and NSARF Study Group. Impact of timing of renal replacement therapy initiation on outcome of septic acute kidney injury. Crit Care. 2011; 15:R134.

44. Wu SC, Fu CY, Lin HH, Chen RJ, Hsieh CH, Wang YC, Yeh CC, Huang HC, Huang JC, Chang YJ. Late initiation of continuous veno-venous hemofiltration therapy is associated with a lower survival rate in surgical critically ill patients with postoperative acute kidney injury. Am Surg. 2012; 78:235-42.

45. Gaudry S, Ricard JD, Leclaire C, Rafat C, Messika J, Bedet A, Regard L, Hajage D, Dreyfuss D. Acute kidney injury in critical care: experience of a conservative strategy. J Crit Care. 2014; 29:1022-27.

46. Tian H, Sun T, Hao D, Wang T, Li Z, Han S, Qi Z, Dong Z, Lv C, Wang X. The optimal timing of continuous renal replacement therapy for patients with sepsis-induced acute kidney injury. Int Urol Nephrol. 2014; 46:2009-14.

47. $\mathrm{Hu} \mathrm{ZJ}$, Liu LX, Zhao CC. [Influence of time of initiation of continuous renal replacement therapy on prognosis of critically ill patients with acute kidney injury]. Zhonghua
Wei Zhong Bing Ji Jiu Yi Xue. 2013; 25:415-19. Influence of time of initiation of continuous renal replacement therapy on prognosis of critically ill patients with acute kidney injury.

48. Kresse S, Schlee H, Deuber HJ, Koall W, Osten B. Influence of renal replacement therapy on outcome of patients with acute renal failure. Kidney Int Suppl. 1999; 56:S75-78.

49. Splendiani G, Mazzarella V, Cipriani S, Pollicita S, Rodio F, Casciani CU. Dialytic treatment of rhabdomyolysisinduced acute renal failure: our experience. Ren Fail. 2001; 23:183-91.

50. Konopka A, Banaszewski M, Wojtkowska I, Stępińska J. Early implementation of continuous venovenous haemodiafiltration improves outcome in patients with heart failure complicated by acute kidney injury. Kardiol Pol. 2011; 69:891-96.

51. do Nascimento GV, Balbi AL, Ponce D, Abrão JM. Early initiation of dialysis: mortality and renal function recovery in acute kidney injury patients. J Bras Nefrol. 2012; 34:337-42.

52. Oh HJ, Shin DH, Lee MJ, Koo HM, Doh FM, Kim HR, Han JH, Park JT, Han SH, Yoo TH, Choi KH, Kang SW. Early initiation of continuous renal replacement therapy improves patient survival in severe progressive septic acute kidney injury. J Crit Care. 2012; 27:743.e9-18.

53. Leite TT, Macedo E, Pereira SM, Bandeira SR, Pontes PH, Garcia AS, Militão FR, Sobrinho IM, Assunção LM, Libório AB. Timing of renal replacement therapy initiation by AKIN classification system. Crit Care. 2013; 17:R62.

54. Yang XM, Tu GW, Gao J, Wang CS, Zhu DM, Shen B, Liu L, Luo Z. A comparison of preemptive versus standard renal replacement therapy for acute kidney injury after cardiac surgery. J Surg Res. 2016; 204:205-12.

55. Oh HJ, Kim MH, Ahn JY, Ku NS, Park JT, Han SH, Choi JY, Han SH, Yoo TH, Song YG, Kang SW, Kim JM. Can early initiation of continuous renal replacement therapy improve patient survival with septic acute kidney injury when enrolled in early goal-directed therapy? J Crit Care. 2016; 35:51-56.

56. Serpytis P, Smigelskaite A, Katliorius R, Griskevicius A. The early use of hemofiltration increases survival in patients with acute renal failure in the setting of intensive cardiac care unit. Glob Heart. 2014; 9:e70. abstract PM046.

57. Perez X, Sabater J, Huguet M, et al. Early timing in septic shock patients. Intensive Care Med. 2012; 38:S263. abstract.

58. Sabater J, Perez XL, Albertos R, Gutierrez D, Labad X. Acute renal failure in septic shock: should we consider different continuous renal replacement therapies on each RIFLE score stage? Intensive Care Med. 2009; 35:S239. abstract 0924.

59. Tsai HW, Yang M, Lin Y, Ko W, Wu K. Outcome in the acute liver failure patients treated with renal replacement 
therapy for acute renal failure: comparison between early or late dialysis. J Am Soc Nephrol. 2005; 16:540A. abstract.

60. Vinsonneau C, Camus C, Combes A, Costa de Beauregard MA, Klouche K, Boulain T, Pallot JL, Chiche JD, Taupin P, Landais P, Dhainaut JF, and Hemodiafe Study Group. Continuous venovenous haemodiafiltration versus intermittent haemodialysis for acute renal failure in patients with multiple-organ dysfunction syndrome: a multicentre randomised trial. Lancet. 2006; 368:379-85.

61. Wald R, Gallagher M, Bagshaw SM. Shedding New Light on an Old Dilemma: Two Trials Examining the Timing of
Renal Replacement Therapy Initiation in Acute Kidney Injury. Am J Kidney Dis. 2017; 69:14-17.

62. Higgins JP, Altman DG, Gøtzsche PC, Jüni P, Moher D, Oxman AD, Savovic J, Schulz KF, Weeks L, Sterne JA, and Cochrane Bias Methods Group, and Cochrane Statistical Methods Group. The Cochrane Collaboration's tool for assessing risk of bias in randomised trials. BMJ. 2011; 343:d5928.

63. Stang A. Critical evaluation of the Newcastle-Ottawa scale for the assessment of the quality of nonrandomized studies in meta-analyses. Eur J Epidemiol. 2010; 25:603-05. 